\title{
Red Temática de Nanociencia y Nanotecnología
}

\author{
Conacyt \\ Dirección Adjunta de Desarrollo Científico y Académico \\ Dirección de Redes
}

Dr. Sergio Fuentes Moyado

Responsable técnico

\section{Comité Técnico Académico de la Red}

Dra. Cecilia Noguez Garrido

Instituto de Física (UNAM)

Dr. Emmanuel Haro Poniatowski

Universidad Autonoma Metropolitana

Dr. Enrique Camps Carvajal

Instituto Nacional de Investigaciones Nucleares

Dr. Gerardo Cabañas Moreno

Instituto Politécnico Nacional

Dr. Jean Philippe Vielle-Calzada

Centro de Investigación y de Estudios Avanzados

Dr. Jesús González Hernández

Centro de Investigación en Materiales Avanzados

Dr. Sergio Fuentes Moyado

Centro de Nanociencias y Nanotecnología (UNAM)

Dr. Humberto Terrones Maldonado

Colaborador

M. C. Edgardo Berea Montes

Farmaquimia

\section{Representante Empresarial}

Ing. Jesús Serrano Landeros

Secretaría de Economía

Representante Gubernamental

\section{INTRODUCCIÓN}

En los últimos 5 años los temas de nanociencias y nanotecnología se han planteado con grandes expectativas debido a su importancia e impacto en una gran cantidad de aplicaciones en diferentes áreas de la industria y la sociedad. Su relevancia es tan grande que se ha dado en llamar como la tercera revolución industrial por su amplia 
variedad de aplicaciones en campos muy diferentes como la medicina, energía y medio ambiente por mencionar sólo algunas.

En el año 2006, el Consejo Nacional de Ciencia y Tecnología (CONACyT) lanzó una Convocatoria para la "Presentación de Ideas para la realización de megaproyectos de investigación científica o tecnológica”. Después de revisar todas las propuestas presentadas de cada megaproyecto en todos los temas, se decidió crear redes temáticas que aglutinaran el esfuerzo de colaboración, apoyos a proyectos y desarrollaran líneas de investigación de acuerdo con las necesidades y oportunidades en temas de interés nacional. Se aprobaron 14 redes y se estableció el objetivo de llegar a 20. En el tema de nanociencias y nanotecnología, las principales propuestas fueron del Centro de Investigación de Materiales Avanzados (CIMAV), del Instituto Potosino de Investigación Científica y Tecnológica (IPICYT), del Laboratorio Nacional en Nanoelectrónica del INAOE y de la Universidad Nacional Autónoma de México (UNAM). En este contexto se creó la Red Temática de Nanociencias y Nanotecnología y se formó un comité técnico académico integrado por los responsables de cada una de las propuestas presentadas al CONACyT.

Un objetivo común de los megaproyectos en nanociencias y nanotecnología fue la creación de una Iniciativa Nacional en Nanotecnología con la finalidad de generar y fortalecer ambas disciplinas con diferentes enfoques, pero todas orientadas a la creación de una mayor capacidad científica y tecnológica en el país y a la búsqueda de impactos económicos, sociales, ambientales y cientificotecnológicos.

Las propuestas consideraban la coordinación de la red o instituciones participantes así como el fortalecimiento de las capacidades a nivel nacional, para poder alcanzar un desarrollo nacional con tecnología propia, que pudiera competir a nivel mundial con productos innovadores. Con excepción de la propuesta del INAOE referida a un campo muy específico de electrónica, el resto de las propuestas presentaron en forma más amplia un campo de aplicaciones muy diverso involucrando una estructura u operación con temas o nodos muy definidos respecto de las oportunidades y campos de investigación que permitían de una forma más ágil la presentación y realización de proyectos de investigación, innovación y colaboración entre las instituciones.

En las líneas de acción se puede apreciar el impacto de la nanotecnología sobre áreas muy diversas; sin embargo, se observan como líneas generales comunes la creación de recursos humanos y posgrados de excelencia, el fortalecimiento de la infraestructura de las redes planteadas así como compartirla, la orientación de proyectos que atiendan las demandas del sector industrial, el trabajo de colaboración interdisciplinaria, el análisis y medición del impacto de la nanotecnología en el medio ambiente y la sociedad, la realización de investigación científica de frontera y la de proyectos de desarrollo tecnológico y de innovación, todo lo anterior permite precisar que el modelo de operación se debe orientar a la definición de proyectos basados tanto en su impacto científico como en la innovación, orientados en temas de interés nacional que sean definidos por el actual Comité de la Red.

En el estudio a nivel nacional acerca de la nanotecnología, publicado en un reporte realizado por el CIMAV para la Secretaría de Economía, se identifica la siguiente tabla de FODA (Fortalezas, Oportunidades, Debilidades, Amenazas:

\section{Fortalezas}

- Infraestructura básica de investigación 
- Masa crítica para investigación y desarrollo en las plataformas tecnológicas con capacidades para su desarrollo en México

- Presencia de empresas manufactureras nacionales con proyectos de nanotecnología en desarrollo

- Acuerdos y tratados internacionales

\section{Oportunidades}

- Incorporación oportuna a la megatendencia de la nanotecnología

- Amplio mercado potencial de productos nanotecnológicos

- Opciones pertinentes para la formación y capacitación de recursos humanos

- Formación de redes nacionales de nanotecnología de carácter incluyente

- Acceso a fuentes internacionales de financiamiento

\section{Debilidades}

- Carencia de un programa o iniciativa nacional de nanotecnología

- Carencia de ámbitos colaborativos entre los diferentes grupos de investigación

- Escaso apoyo gubernamental a la CyT

- Inexistencia de un modelo de transferencia del conocimiento a la sociedad

- Falta de cultura empresarial en inversiones de riesgo

- Falta de equipo científico de vanguardia y de plantas piloto

- Excesivos trámites y burocracia pesada

- Desconocimiento generalizado del tema de la nanotecnología

\section{Amenazas}

- Ampliación de la brecha tecnológica con respecto a países más avanzados

- Desconocimiento de riesgos ambientales, en la seguridad y en la economía

- Pérdida de ventajas competitivas ante países como China, India, Singapur, etc.

- Crisis financiera en el ámbito internacional

Con base en este análisis es posible proponer una serie de estrategias y acciones tendientes a impulsar el área de $\mathrm{NyN}$ en el país.

\section{Organización de LAS Redes temáticas}

A través de la Dirección de Redes se llevó a cabo el proceso de creación de las redes temáticas, considerando, como ya se mencionó anteriormente, las propuestas surgidas de la convocatoria de megaproyectos. A continuación se describen los objetivos, organización, funciones y atributos de las redes

\section{Objetivos del programa de redes temáticas}

1. Promover y fortalecer la construcción y desarrollo de redes científicas nacionales en temas estratégicos que respondan a problemas científicos, tecnológicos y sociales y procuren la vinculación academia-empresa. 
2. Alcanzar soluciones articuladas con enfoque multidisciplinario y multiinstitucional, y estructuradas de manera que contribuyan al desarrollo nacional y al bienestar de la población.

\section{Objetivos específicos de las redes}

1. Elaboración de un Proyecto Nacional de desarrollo de la investigación científica y tecnológica en el tema.

2. "Estado del arte" en México en el tema de la Red.

3. Un catálogo de recursos humanos e infraestructura en México.

4. Financiamiento de fuentes nacionales y extranjeras.

5. Proyectos susceptibles de vinculación con el sector público y privado.

6. Proyectos académicos multiinstitucionales viables en ciencia básica u orientada

7. Proyectos orientados a resolver - o crear las condiciones para hacerlo- problemas estratégicos de la sociedad mexicana.

\section{Organización propuesta}

A continuación se muestra el diagrama con la organización de la propuesta hecha por el CONACyT para la operación de las redes temáticas:

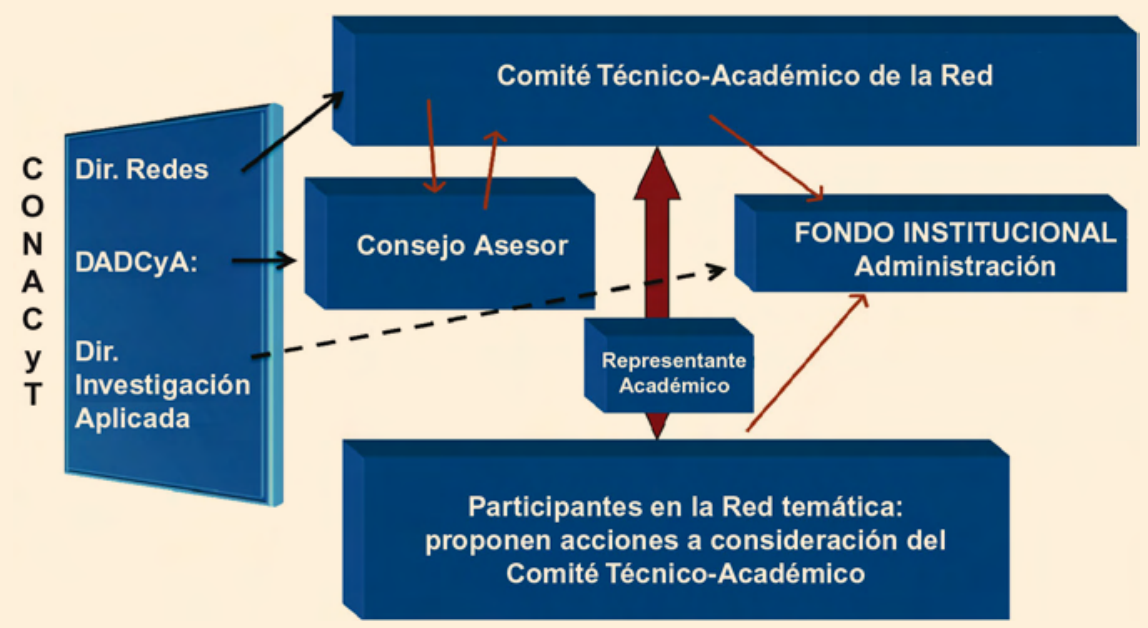

Para la realización y obtención de los objetivos tanto generales como específicos se propone una organización matricial con la siguiente estructura:

1. Un comité técnico académico. Órgano colegiado para la toma de decisiones de la red temática (1 por cada red). Conformado por representantes de grupos de investigación destacados (hasta 10), el director de redes temáticas, un representante del sector técnico-empresarial, un representante del sector gubernamental, un académico distinguido. 
2. Un consejo asesor. Instancia de evaluación y seguimiento del desempeño de las redes temáticas. Conformado por tres representantes institucionales (IES), un representante del sector técnico-empresarial, dos distinguidos académicos, un representante de las áreas sustantivas del CONACyT, dos representantes de la Junta de Gobierno del CONACyT, el director adjunto de desarrollo científico y académico como coordinador.

3. Un representante académico de la red designado por el comité académico.

4. Despacho externo que llevará el control de las ministraciones, pagos y comprobaciones de cada gasto de las redes.

5. Investigadores participantes de cada red temática de reconocido prestigio en el tema y que lleve a cabo proyectos que tengan compatibilidad temática.

\section{Funciones y atributos}

1. Comité técnico académico:

a. Elegir al representante del comité técnico académico de la red.

b. Aprobar la integración de nuevos miembros de la red.

c. Elaborar y aprobar:

a) Programa general de trabajo de la red

b) Presupuesto ejecutivo

d. Desarrollar manual interno de la red (normas operativas).

e. Determinar acciones y proyectos a realizar por la red (a propuesta de los miembros de la red).

f. Rendir los informes técnicos.

g. Calendario de ministraciones

h. Nueva red o cancelación de la existente.

i. Faculta al representante académico

2. Consejo asesor:

a. Evalúa y aprueba el programa general de trabajo de la red y el presupuesto Ejecutivo de cada red a propuesta del comité técnico-académico.

b. Evalúa el desempeño de la red para determinar su continuidad.

c. Aprueba la creación de nuevas redes.

d. Propone programas y presupuesto.

e. Observaciones y recomendaciones de la operación de la red.

3. Representante académico:

a. Representar los intereses de la red temática ante CONACyT y dentro del fideicomiso.

b. Mantener la comunicación entre la red y CONACyT

c. Suscribir el convenio de asignación de recursos.

d. Instruir la dispersión de recursos a los investigadores de la red.

e. Convocar a las sesiones del comité técnico académico. 

f. Entregar los informes técnico-financieros
g. Llevar el seguimiento de acuerdos del comité técnico académico.
h. Presenta proyectos e integrantes de la red.
i. Desarrolla el manual interno de la red.

4. Despacho externo
a. Documentos fiscales a nombre del FOINS.
b. Elegibilidad.
c. Determinación de diferencias.

5. Investigadores participantes en la red.

Habrá tantos investigadores como sea posible de acuerdo con los criterios de aceptación y pertinencia tanto de su reconocimiento y prestigio como de los proyectos de investigación tengan compatibilidad y pertinencia con los objetivos de la red. El investigador hará personalmente la comprobación de los recursos a través de un sistema diseñado e instalado en su computadora. También hará la presentación de los resultados del mismo.

Esta forma de operación ya está aprobada y sólo es cuestión de tiempo para que salgan las convocatorias y que se pueda implementar en cada una de las redes temáticas por lo que con esta base se propone el manual de operación sin que éste entre en conflicto con lo que ya está aprobado y listo para ser puesto en marcha en los meses de febrero o marzo del 2009.

Un cambio significativo respecto a otras formas de operación y administración es que la participación de los investigadores es a título personal y no con base en el apoyo de una institución, si bien ésta última continuará respaldando al investigador, no será la primera responsable en el gasto y seguimiento de los proyectos ni tampoco de la evaluación de los proyectos que el investigador realice al interior de cada red. Otro cambio es la administración externa de los recursos ya que no se utilizará la infraestructura de las instituciones en la que colabora el investigador, lo que se busca es agilizar el proceso de entrega de recursos, comprobación de gastos y hacer más rápida y eficiente la evaluación de los proyectos para que los recursos no se detengan y se pueda continuar con la realización de los proyectos.

Aunque está mencionado como parte de los objetivos específicos en realidad con la organización, como se propone, falta contar con un puente de vinculación más dedicado en tiempo y forma para poder contar en forma eficiente y rápida con retroalimentación de los sectores gubernamental y empresarial y al mismo tiempo sirva como brazo de inteligencia estratégica a la red.

\section{RED TEMÁTICA dE NANOCIENCIAS Y NANOTECNOLOGIA}

Objetivos, metas, estrategias, acciones y entregables

Debido a que en México la nanociencia y la nanotecnología ( $\mathrm{NyN}$ ) es un área emergente con gran potencial que todavía no cuenta con una masa crítica en el país que pueda tener impacto nacional, se deben unir fuerzas entre varias instituciones y sectores con la finalidad de establecer una red de excelencia que aproveche las fortalezas 
y reduzca las debilidades para impactar en la sociedad y el sector productivo. Esta red pretende lograr los siguientes objetivos:

\section{Objetivo 1}

Efectuar estudios diagnósticos que presenten el "estado del arte", los retos y las oportunidades existentes en México, en materia de la temática de la red.

Este objetivo busca actualizar y complementar los estudios y diagnósticos ya existentes sobre el estado del arte de NyN, considerando los retos más importantes y los nichos u oportunidades en los que nuestro país puede competir.

META 1: Actualizar los estudios de capacidades en recursos humanos e infraestructura

Se cuenta con información inicial sobre recursos humanos e infraestructura que estará a disposición de la red. Sin embargo, ésta se debe actualizar y enfocar a las líneas de investigación en que la red pueda ser más fuerte, tenga mayor impacto y refleje el estado actual del tema.

META 2: Fortalecer de manera importante a la red a través de una estrategia que permita al país incursionar en nanobiotecnología.

Los estudios previos que se tienen van enfocados a la parte de materiales inorgánicos, con la participación del CINVESTAV-Irapuato, se aporta su experiencia en la parte biológica y se enriquece a la red. Es un hecho que la actividad multidisciplinaria en nanobiotecnología es un área en la que se están realizando proyectos de gran impacto de nivel internacional

\section{Estrategias}

Aunque ya se cuenta con una base de datos que indican las capacidades académicas en relación a recursos humanos y equipos de laboratorio, se realizará la actualización del inventario de capacidades nacionales en el tema de nanociencias y nanotecnología, el cual deberá incluir:

- Conocer los recursos humanos capacitados, tomando en cuenta el grado obtenido

- Determinar la infraestructura experimental utilizada en actividades relacionadas con el tema

- Censar la formación de recursos humanos a nivel de grado y de posgrado. Se incluirán los diferentes programas nacionales por institución

\section{Acciones}

- Primeramente, se redactará un cuestionario que identifique las instituciones y las empresas nacionales que realizan o planean realizar actividades en el tema de nanociencias y nanotecnología. A través del cuestionario se deberá obtener información sobre las capacidades existentes y humanas. Se solicitará información a instituciones como: UNAM, IPN, Sistema de Centros CONACyT, UAM, centros sectorizados (CENAM, IIE, IMP, ININ), universidades e institutos públicos y privados. 
- Se identificarán los programas de grado y posgrado que formen recursos en el tema de nanociencias y nanotecnología, registrando las diversas modalidades, esto es, programas dedicados al tema o aquellos que ofrecen otras alternativas como opciones terminales o que incluyen materias en el programa sobre el tema en su currículum. Será importante conocer las temáticas en las cuales los estudiantes de posgrado están realizando su trabajo de tesis.

- Se identificarán los laboratorios, por institución, que realicen actividades en nanociencias y nanotecnología, esto con el fin de determinar la infraestructura experimental (equipos, plantas piloto, capacidades de cómputo, etc.) con que cuentan las instituciones académicas y empresas. Con esta información se obtendrá el inventario de capacidades experimentales y así poder ofertarlo a la comunidad.

- Se identificarán las líneas de investigación y los proyectos que actualmente se están realizando en los diferentes laboratorios, incluyendo la fuente de financiamiento. Esto nos ayudará a reconocer las áreas en las que tenemos nuestras competencias clave, así como el diseño de las plataformas científico tecnológicas con mayor potencial en el entorno global.

- A través de los investigadores del área de biotecnología se realizarán los estudios de prospectiva de colaboración entre la parte biológica y la de nanomateriales.

En los estudios y diagnósticos sobre el estado del arte incluiremos el impacto ambiental y en seres vivos. Se contribuirá a implementar los acuerdos de estándares internacionales en colaboración con el Centro Nacional de Metrología (CENAM) y los grupos encargados en la OECD y el Instituto Nacional de Ecología.

Es importante mencionar que los dos laboratorios nacionales en NyN apoyados por el CONACyT con fondos concurrentes, participan en la red (IPICyT y CIMAV), por lo que infraestructura de punta con un costo aproximado de 80 millones de pesos estará disponible para la red, así como infraestructura ya existente en las instituciones participantes. Por otro lado, el IPICyT cuenta con un posgrado en NyN que podrá ser el inicio de un posgrado nacional en el campo.

\section{Entregables}

- Documento del estado del arte en en nanociencias y nanotecnología, identificando fortalezas, oportunidades, desafíos y amenazas.

- Análisis de la Comisión Técnico Académica acerca de cuáles son los retos y las oportunidades existentes en nanociencias y nanotecnología, así como las recomendaciones para que México pueda ser competitivo en el área.

\section{Objetivo 2}

Obtener un catálogo de recursos humanos en nanociencias y nanotecnología de México.

Se cuenta con información inicial sobre recursos humanos que estará a disposición de la red. Sin embargo, ésta se debe actualizar y enfocar a las líneas de investigación en que la red pueda ser más fuerte y tener mayor impacto. 


\section{Estrategia}

- Conocer los recursos humanos capacitados en las instituciones de educación superior del país.

- Determinar la infraestructura experimental disponible para los estudiantes involucrados en investigaciones relacionadas con el tema.

- Censar la capacidad de formación de recursos humanos a nivel de grado y de posgrado.

\section{Entregables}

Consideramos que los puntos anteriores estarán terminados en un plazo de seis meses y constituirán parte importante de la justificación de la red. Sin embargo, hay que decir que esta información es dinámica y se actualizará regularmente en la página electrónica de la red temática NyN.

- Un catálogo de programas de formación de recursos humanos identificando las potencialidades de cada región.

\section{OBjetIVo 3}

Análisis de proyectos académicos multi institucionales en ciencia básica u orientada de interés e importancia nacional argumentando y sustentando su viabilidad.

Se identificarán áreas estratégicas para generar proyectos multidisciplinarios, que aporten soluciones integrales en el entendimiento de la nanotecnología y sus posibles aplicaciones. Se buscará conjugar la experiencia de diversos grupos para la resolución de tópicos o áreas de interés primordial para la innovación tecnológica.

META: Establecer programas que fomenten las actividades científicas que incrementen el conocimiento.

Se promoverá la generación de proyectos de ciencia básica, que busquen incrementar el conocimiento de los fenómenos a la nanoescala y que puedan generar en el mediano o largo plazo, nuevas tecnologías que incrementen la competitividad económica o beneficien a la sociedad.

Se promoverá la investigación en campos multidisciplinarios, a través de redes y macroproyectos, incluyendo aportaciones de tipo químico, físico, biológico, así como de ingeniería y materiales.

\section{Estrategias}

- Identificar áreas estratégicas y las capacidades del país para generar proyectos de investigación básica, que generen conocimiento y conceptos innovadores en el estudio de propiedades, procesos, estructuras, arquitecturas, simulación así como técnicas de alta resolución especial y temporal.

\section{Acciones}

- Proponer proyectos de nanociencia y nanotecnología, en las modalidades de proyectos individuales y de grupo. 
- Proponer la creación de infraestructura de caracterización a la nanoescala en regiones específicas del país.

- Crear subredes de nanociencia a nivel nacional.

- Establecer un programa de entrenamiento y capacitación en el extranjero del personal técnico que va a operar los equipos de alta tecnología.

- Favorecer el intercambio académico a nivel nacional e internacional.

\section{Entregables}

- Documento de áreas potenciales de desarrollo en nanociencias y nanotecnología, identificando fortalezas, oportunidades, desafíos y amenazas.

- Recomendación de la Comisión Técnico Académica acerca de cuáles son los campos en nanociencias y nanotecnología en los que México puede ser competitivo.

- Propuesta de formación de subredes de nanociencia entre los principales grupos de investigación en el país.

- Reportes de avances de las reuniones temáticas.

- Programas de cursos formativos y de entrenamiento para las universidades e industria.

- Reporte de acciones de intercambio académico internacional.

\section{OBJETIVo 4}

Diseño y ejecución de proyectos en ciencia aplicada susceptibles de lograr la vinculación con el sector público y privado. Además de buscar financiamiento de fuentes tanto nacionales como extranjeras, se dará prioridad a los proyectos que permitan esquemas ejecutables y que consideren la solución de problemas reales de la sociedad mexicana.

Este objetivo busca detectar las posibles áreas de vinculación entre los grupos de investigación y las entidades gubernamentales o empresariales que requieren de resolver problemas o innovar sus procesos basados en el uso de nanociencias y nanotecnología. Una vez identificados los aspectos de interés común se procederá a armar proyectos de índole tecnológica que contemplen la aplicación, transferencia y/o producción de materiales o sistemas.

META 1: Transferir los avances tecnológicos en productos que incrementen la mano de obra, el crecimiento económico y el bienestar de la sociedad.

La nanotecnología ha pasado de ser una promesa a una serie de realizaciones que empiezan a tener impacto en la manera diaria de vivir. Se han desarrollado productos innovadores en muchas áreas, como textiles, cosméticos, transporte, alimentos y probablemente los de mayor impacto en medicina. También se esperan grandes avances en computación.

\section{Estrategias}

- Identificar las capacidades del país en investigación científica aplicada e investigación tecnológica.

- Promover la interacción de los grupos de investigación con la industria. 
o Organizar foros de intercambio de ideas entre ambos grupos.

- Establecer redes de colaboración entre grupos de investigación e industria.

o Establecer grupos de trabajo conjunto (investigación-industria) en los institutos, centros y laboratorios nacionales.

o Promover el uso de las instancias y programas del CONACyT para el financiamiento de proyectos a empresas.

- Buscar financiamiento para los proyectos estratégicos dirigidos a resolver problemas relacionados con las áreas en las que México requiere incrementar su capacidad de innovación.

- Promover las capacidades de innovación que se pueden lograr a través de la nanotecnología en el sector privado y los tres niveles de gobierno, para incrementar las posibilidades de financiamiento y desarrollo de los programas y proyectos.

\section{Acciones}

PROMOCIÓN

- Establecer un programa de incentivos al desarrollo tecnológico en la industria y los laboratorios de investigación.

- Mantener canales de comunicación e información abiertos que promuevan la incorporación de nuevas empresas a la iniciativa.

- Apoyar a las universidades estatales para que se incorporen a los proyectos estratégicos.

- Favorecer el intercambio de recursos humanos entre la universidad y la industria.

\section{PROYECTOS ESTRATÉGICOS}

- Proponer proyectos estratégicos, en las modalidades de proyectos de grupo.

- Proponer la creación de infraestructura para los Proyectos Estratégicos en regiones específicas del país.

- Proponer equipamiento dedicado al estudio de las principales enfermedades que afectan a los mexicanos.

- Proponer un programa de entrenamiento y capacitación en el extranjero del personal técnico que va a operar los equipos de alta tecnología.

- Favorecer el intercambio académico a nivel nacional e internacional.

META 2: Desarrollo sustentable y ético de la nanotecnología

Se deberá establecer que todas las acciones comprendidas en esta iniciativa que buscan crear desarrollos en nanotecnología, incrementar la competitividad, proporcionar mayor valor agregado y al mismo tiempo aumentar la oferta de empleo y los ingresos de los trabajadores, que se realicen con un estricto apego a la conservación del medio ambiente y a un desarrollo ético de la nanotecnología. Asimismo, se debe considerar una meta importante el mantener al público informado de las repercusiones de la nanotecnología y promover su aceptación. 


\section{Estrategias}

- Promover el contacto entre las agencias gubernamentales reguladoras, los investigadores y la industria.

- Proteger que los desarrollos en nanotecnología se encuentren en el marco jurídico adecuado.

- Estudiar los riesgos potenciales de los nanomateriales.

- Establecer las normas y estándares relacionados con el uso de los productos y procesos nanotecnológicos.

- Promover reuniones entre autoridades del medio ambiente e investigadores para evaluar riesgos ambientales.

- Adherirse a los acuerdos internacionales sobre desarrollo responsable de la nanotecnología.

- Establecer el ciclo nacimiento-vida-muerte de los productos nanotecnológicos para poder disponer ecológicamente de ellos.

- Analizar el impacto de la nanotecnología en el crecimiento económico, el estándar de vida y la competitividad.

- Incluir cursos sobre desarrollo sustentable de la nanotecnología en licenciatura y posgrado.

- Reglamentar los experimentos con seres vivos.

Se analizarán los acuerdos de estándares internacionales en colaboración con el Centro Nacional de Metrología (CENAM) y los grupos encargados en la OECD y en el Instituto Nacional de Ecología.

\section{OBjetivo 5}

Conjuntar la información generada por los estudios diagnósticos que identifiquen los retos y las oportunidades existentes en México, en un Proyecto Nacional.

Este objetivo busca generar un proyecto ejecutivo del estado del arte de NyN, considerando los retos más importantes y los nichos u oportunidades en los que nuestro país pueda competir.

META: Generar un documento ejecutivo que contenga los planes de desarrollo a nivel científico, tecnológico, desarrollo y capacitación de recursos humanos e infraestructura.

\section{Entregables}

- Proyecto ejecutivo nacional en nanociencias y nanotecnologia, conteniendo las recomendaciones de expertos en el área y las acciones que se deberán realizar para que México pueda ser competitivo en el área.

Además de los objetivos específicos identificados se incluirán otras acciones muy relevantes en el tema.

\section{Acciones en medio ambiente y salud}

- Financiar investigaciones de los efectos de la nanotecnología en el medio ambiente y la salud. 
- Financiar investigaciones sobre el ciclo de vida de los nanomateriales y el reciclado.

- Financiar investigaciones que evalúen el riesgo a la salud, durante la producción y el manejo de nanomateriales en las investigaciones, el escalamiento y la producción.

- Establecer reglas y manuales de uso para trabajar con nanomateriales.

- Difundir en todos los niveles las normas de seguridad.

\section{Acciones en comunicación}

- Fomentar la comunicación y el diálogo con el público a través de televisión, museos, exposiciones, etcétera.

- Crear materiales enfocados a divulgar los avances en nanotecnología.

- Hacer encuestas sobre la percepción del público.

- Detectar los obstáculos al desarrollo nanotecnológico y la manera de superarlos.

- Fomentar el desarrollo ético de la nanotecnología.

- Determinar los efectos a corto, mediano y largo plazo de la nanotecnología.

- Informar a la ciudadanía sobre los avances en nanotecnología para incrementar la aceptación de los procesos.

\section{VALORES Y PRINCIPIOS GUÍA DE LA RED}

El integrarse en una red temática conlleva múltiples ventajas, siendo la principal el trabajo coordinado, lo cual optimiza las capacidades humanas y físicas existentes en México. Esto permitirá abordar las temáticas identificadas como de mayor oportunidad con una amplia posibilidad de éxito, tanto en el ámbito académico como en el tecnológico.

Por lo anterior, se propone un modelo orientado hacia:

- El desarrollo de investigación científica básica

- El desarrollo de proyectos con base en las líneas de investigación definidas en cada área temática y proceso crítico en la red temática.

- El desarrollo de proyectos de investigación y desarrollo identificados a partir de necesidades detectadas en el mercado nacional o internacional.

- La formación de recursos humanos con la creación de un mayor número de programas de doctorado y maestría relacionados con la nanotecnología, así como el fortalecimiento y especialización de los existentes.

- El fortalecimiento de cooperaciones con instituciones expertas y reconocidas a nivel mundial en nanociencias y nanotecnología y que permita fortalecer la infraestructura humana y física para el desarrollo en México.

Lo anterior permitirá un seguimiento continuo y un enfoque dirigido hacia la obtención de resultados concretos.

Listado de valores y principios guía

1. Fortalecer y fomentar la colaboración entre los miembros de la red de nanociencias y nanotecnología. 
- Trabajo en equipo, multidisciplinario y orientado a resultados.

- Generación de plataformas efectivas de comunicación que fortalezcan la colaboración de los miembros de la red y aseguren su operación.

- Programas de colaboración de interés común para los miembros de la industria y la academia que cuente con mecanismos para el desarrollo de investigaciones conjuntas que permitan el flujo de conocimiento y capacidades entre sus miembros.

- Trabajo colaborativo con el sector productivo y gubernamental, que permita la detección de grandes temas nacionales para el desarrollo de proyectos de investigación e innovación en el tema de Nanociencias y Nanotecnología.

2. Desarrollo y fortalecimiento de capacidades para formar recursos humanos de calidad internacional.

- Fortalecimiento y creación de programas de posgrado de calidad internacional en instituciones educativas mexicanas.

- Programa con orientación a fortalecer las capacidades científicas y tecnológicas en nanociencias y nanotecnología para formar recursos humanos de calidad internacional.

- Fortalecimiento de las plantillas de profesores (repatriación, intercambios, estancias, entre otros).

3. Fortalecimiento de las actividades de investigación, desarrollo tecnológico e innovación basadas en nanociencias y nanotecnología.

- Definición de líneas de investigación aplicada, desarrollo tecnológico e innovación en nanociencias y nanotecnología.

- Apoyo a las iniciativas de investigación y desarrollo precompetitivo para los sectores y nichos de oportunidad que sean definidos como estratégicos para la red.

- Desarrollo de productos innovadores que sean llevados hasta la etapa de comercialización

4. Utilización de infraestructura de investigación, de manera compartida por los miembros de la red.

- Fortalecimiento de la infraestructura existente y puesta en servicio

- Definición de mecanismos de colaboración y operación de la red de Laboratorios de innovación de nanociencias y nanotecnología.

- Actualización de equipos y modernización de procesos

5. Trabajo de inteligencia estratégica para definir sectores industriales y nichos de oportunidad en nanociencias y nanotecnología con posibilidad de generar impacto en México en 5 años.

- Desarrollo de metodologías y herramientas para actividades de inteligencia y vigilancia tecnológica en nanociencias y nanotecnología. 
- Prospectiva tecnológica para el análisis y desarrollo de mapas tecnológicos y estudio de arte con visión global para identificar sectores industriales clave, nichos de oportunidad, líneas de desarrollo y nuevos productos.

6. Facilitar la participación de los sectores industriales y gubernamentales estratégicos en México que se puedan beneficiar con el uso de nanociencias y nanotecnología.

- Proyectos de alto contenido científico tecnológico y de impacto en los sectores industriales identificados como de mayor oportunidad para la nanociencias y la nanotecnología.

7. Fomentar la cultura de la propiedad intelectual.

- Capacitación de miembros de la red en temas de propiedad intelectual.

- Esquemas de colaboración en proyectos conjuntos para la generación de propiedad intelectual (patentes y derechos de autor).

8. Propiciar la colaboración internacional de calidad para fortalecer la red.

- Establecer alianzas estratégicas con expertos de instituciones internacionales líderes en nanociencias y nanotecnología.

- Vinculación efectiva y transparente entre instituciones nacionales e internacionales.

9. Programas efectivos de promoción y difusión de las actividades y logros de la red.

- Promoción del programa de nanociencias y nanotecnología a nivel nacional e internacional, a través de talleres, seminarios, congresos, eventos, relacionados con el tema.

- Desarrollo de herramientas efectivas de difusión y promoción de la red.

10. Enfoque operativo.

- Compromiso de participantes de la red en la elaboración de proyecto alineados a los temas o nodos que defina la red.

- Integración de comités ad hoc para las diversas actividades de la red.

- Indicadores de impacto cuantitativo y cualitativo de las iniciativas de la red.

- Asignación de recursos a los beneficiarios a través de procesos de calidad, factibilidad y pertinencia.

- Actividades de la red orientadas a crear valor.

11. Implementación de acciones que permitan el financiamiento nacional e internacional. 
- Identificar fuentes de financiamiento en la red de nanociencias y nanotecnología. Pueden provenir del sector privado, gubernamental (federal y estatal) o internacional.

12. Propuestas para mejoras de políticas científico tecnológicas que impulsen el desarrollo y uso de nanociencias y nanotecnología en México

- Crear visión nacional y regional para impulsar y promover iniciativas en el campo de las nanociencias y la nanotecnología.

\section{Organización}

\section{a. Propuesta}

Con base en la propuesta ya aprobada del Conacyt se presenta la siguiente organización para la red de nanociencias y nanotecnología, en la que se añade el área de inteligencia estratégica y casos de negocio para poder apoyar en forma más estrecha la colaboración de la red con los sectores empresariales y gubernamentales.

Para la operación y manejo de la red se propone una organización matricial con la siguiente estructura:

1. Un comité técnico académico (CTA). Órgano colegiado para la toma de decisiones de la red temática (1 por cada red). Conformado de acuerdo con lo establecido en las reglas de Conacyt.

2. Un consejo asesor. Instancia de evaluación y seguimiento del desempeño de las redes temáticas. Conformado de acuerdo con lo establecido en la reglas de Conacyt. El consejo asesor es una instancia superior por arriba del CTA y es común a todas las redes.

3. Un representante académico de la red designado por el comité técnico académico.

4. Un área de inteligencia estratégica y casos de negocio conformado de acuerdo a los lineamientos del representante académico y el comité técnico académico. Se propone a una empresa externa para coordinar las actividades con reconocido prestigio en el tema y con conocimientos en inteligencia estratégica y casos de negocio.

5. Despacho externo que llevará el control de ministraciones, pagos y comprobaciones de cada gasto de las redes.

6. Investigadores participantes de cada red temática de reconocido prestigio en el tema y que lleve a cabo proyectos que tengan compatibilidad temática.

Se recomiendan que se integren como punto de partida para los trabajos de la red nodos o competencias claves para la presentación y operación de los proyectos

Se recomienda que se haga una clasificación por aplicación y relevancia en el entorno nacional.

Cada tema o nodo contará con una matriz con la información de las instituciones que participan en esa línea de investigación y el nombre de los investigadores, personal técnico que participa y los laboratorios y equipos que se están utilizando. 
b. Roles y responsabilidades

COMITÉ TÉCNICO ACADÉMICO

a. Elige al representante del comité técnico académico de la red.

b. Aprueba la integración de nuevos miembros de la red.

c. Elabora y aprueba:

a) Programa general de trabajo de la red.

b) Presupuesto ejecutivo.

d. Desarrolla el manual interno de la red (normas operativas).

e. Determina acciones y proyectos a realizar por la red (a propuesta de los miembros de la red).

f. Rinde los informes técnicos.

g. Propone el calendario de ministraciones.

h. Faculta al representante académico.

i. Participa en la presentación de resultados de las diferentes instituciones y los líderes de proyecto en una reunión anual.

j. Aprueba, supervisa, evalua y asegura el cumplimiento de las funciones del representante académico de la red y el consejo asesor.

k. Difunde las actividades y los logros de la red al público.

CONSEJO ASESOR

a. Evalúa y aprueba el programa general de trabajo de la red y el presupuesto Ejecutivo de cada red a propuesta del comité técnico-académico.

b. Evalúa el desempeño de la red para determinar su continuidad.

c. Aprueba la creación de nuevas redes.

d. Propone programas y presupuesto.

e. Emite observaciones y recomendaciones de la operación de la red.

f. Fortalece la vinculación a nivel internacional y fuentes de financiamiento para proyectos de la red.

\section{REPRESENTANTE ACADÉMICO}

a. Representa los intereses de la red temática ante Conacyt y dentro del fideicomiso.

b. Mantiene la comunicación entre la red y Conacyt.

c. Suscribe el convenio de asignación de recursos.

d. Instruye la dispersión de recursos a los investigadores de la red.

e. Convoca a las sesiones del comité técnico académico.

f. Entrega los informes técnico-financieros.

g. Lleva el seguimiento de acuerdos del comité técnico académico.

h. Presenta proyectos e integrantes de la red.

i. Desarrolla el manual interno de la red.

j. Revisa las políticas de propiedad intelectual generadas para la adecuada explotación del conocimiento generado con los proyectos de I+D.

k. Propone los requerimientos para la generación de posgrados de calidad internacional y el reforzamiento de los ya existentes. 
1. Presenta los resultados y los logros alcanzados ante instituciones como el Conacyt y el público en general.

$\mathrm{m}$. Establece convenios con las instituciones nacionales e internacionales.

n. Establece el calendario de reuniones con el consejo asesor.

o. Coordina la presentación de los logros alcanzados una vez por año al comité técnico académico.

p. Canaliza los proyectos detectados por el área de inteligencia estratégica y establece los acuerdos necesarios con el sector productivo para la realización de proyectos y su implementación.

q. Establece una comunicación continua entre el sector productivo y la red.

r. Genera los lineamientos para política de la protección intelectual.

s. Propone la realización de estudio de mercado para detectar las oportunidades y necesidades en las empresas.

ÁREA DE INTELIGENCIA ESTRATÉGICA

a. Determina los estudios de patentes, artículos y en general mantener un constante monitoreo de la información generada a nivel mundial sobre los avances en el área de nanociencias y nanotecnología.

b. Detecta las oportunidades de mercado en conjunto con los investigadores.

c. Revisa y dar seguimiento al plan estratégico a 5 años de la red de acuerdo con los temas de interés nacional y las nuevas oportunidades que se detecten en el mercado y las tendencias a nivel mundial.

d. Prepara la información al representante académico para presentar la rendición de cuentas del proyecto.

e. Prepara los logros y los impactos al Comité Técnico Académico para presentar la información al público y a las autoridades competentes.

f. integra la información completa de cada proyecto que se realiza en la red su implicación y el tipo de propiedad intelectual que se puede generar.

g. Construye una política de transferencia de tecnología para los proyectos y sus resultados, que permita la transferencia a empresas o aquellas interesadas en un proyecto específico.

h. Integra los casos de negocio que se puedan generar con los proyectos de I+D incluyendo plan de negocio, requerimientos de inversión y fuentes de financiamiento.

i. Presenta los proyectos en conjunto con cada investigador a posibles empresas interesadas en la tecnología que se desarrolló.

j. Implementa la política de propiedad intelectual para la transferencia tecnológica y comercialización de los proyectos de I+D.

k. Integra las soluciones requeridas para llevar hasta la aplicación comercial los casos de negocio exitosos.

\section{Despacho externo}

a. Recaba documentos fiscales a nombre del FOINS.

b. Determina la elegibilidad.

c. Determina las diferencias.

d. Revisa los montos del presupuesto autorizado para cada proyecto. 
e. Da seguimiento al gasto corriente y en inversión de la red.

f. Hace la entrega del presupuesto a cada proyecto de investigación aprobado por el comité técnico académico.

g. Lleva el control administrativo y financiero de la red.

\section{INVESTIGADORES PARTICIPANTES EN LA RED}

c. Mantienen estrecha vinculación con otros investigadores de la red.

d. Mantienen la comunicación con el representante académico.

e. Presentan la información de cada proyecto de I+D ante el comité técnico académico.

f. Hacen un levantamiento general de los recursos tanto humanos como en infraestructura que aplica en su proyecto.

g. Coordinan las actividades de los investigadores y personal a su cargo para la realización del proyecto.

h. Presentan nuevos proyectos en forma conjunta con el área de inteligencia estratégica o en forma individual así como los avances de los proyectos ante el consejo asesor, representante académico y el comité técnico académico.

i. Realizan reuniones de avance de proyectos con el personal que participa en el mismo.

j. Presentan ante el representante académico la información que se requiera para la preparación de los reportes.

k. Revisan en forma conjunta con el área de inteligencia estratégica los artículos o patentes generados, para evaluar el camino a seguir en su publicación.

l. Presentan en la reuniones con el comité técnico académico y elcConsejo asesor los avances y logros del proyecto de I+D a su cargo.

m. Generan necesidades para la formación de recursos humanos necesarios para la consecución de los proyectos tanto de investigación básica, aplicada, o de equipo y proceso, como de los productos definidos a ser desarrollados por la red.

n. Colaboran en la evaluación de los nuevos casos de negocio generados en cada producto que se desarrolló mediante I+D e Innovación.

o. Programan el presupuesto asignado a su proyecto de desarrollo.

p. Presentan la comprobación de los recursos al Despacho externo para su revisión.

\section{c. Integración de participantes a la Red}

\section{COMITÉ TÉCNICO ACADÉMICO}

Estará integrado inicialmente por un representante de cada una de las partes integrantes o promotores de la red, pudiendo llegar a tener un máximo de 10 miembros, los cuales deberán pertenecer a sectores académicos y el director de redes temáticas, un representante del sector técnico empresarial, un representante del sector gubernamental, un académico distinguido.

De entre ellos, habrá un representante académico, elegido unánimemente o por simple mayoría de votos, y quien asumirá la obligación de representar a la red por convocatoria, sin perjuicio de que los demás miembros lo hagan y tendrá voto de calidad para el caso de empate en las decisiones que se tomen, además de las facultades que deriven de este pacto. 
El comité quedará integrado en un plazo no mayor de tres meses, a partir de la firma del convenio o acuerdos de formación de la red.

Los cargos desempeñados en el comité técnico académico serán de carácter honorífico y no recibirán retribución económica o en especie por la ejecución de dichos cargos.

\section{REPRESENTANTE ACADÉMICO}

El representante académico será quien funja como el encargado de presentar los proyectos en la red, entre otras responsabilidades mencionadas anteriormente, será electo por el comité técnico académico y será remplazado cada dos años de su función o en su caso el comité técnico puede solicitarle la extensión por un máximo de un año.

El representante académico sólo puede ser electo de entre los representantes de las instituciones promotoras para la creación de la red.

A su vez el representante académico podrá contar con el apoyo de personal que lo auxilie en su labor al interior de la red.

\section{CONSEJO DE ASESORES}

El consejo de asesores será conformado por tres representantes institucionales (IES), un representante del sector técnico empresarial, dos distinguidos académicos, un representante de las áreas sustantivas del CONACyT, dos representantes de la Junta de Gobierno del CONACyT, el director adjunto de Desarrollo Científico y Académico como Coordinador en forma adicional y por acuerdo de los miembros del consejo asesor se podrá invitar hasta un máximo de dos distinguidos académicos de instituciones internacionales para promover la vinculación y trabajo conjunto con organismos internacionales.

El cargo de los consejeros asesores de la red serán de carácter honorífico y por tanto no recibirán retribución económica o en especie por la ejecución de dichos cargos.

El periodo como consejero no será mayor de tres años y en cualquier momento se puede presentar la renuncia y analizar el ingreso de un nuevo consejero por parte del comité técnico académico en sus sesiones normales de operación.

\section{INTELIGENCIA ESTRATÉGICA}

La integración del área de inteligencia estratégica se puede estructurar de la siguiente forma:

1. Con el apoyo externo de una empresa con reconocido prestigio en el tema de vigilancia tecnológica, estudios de arte, vinculación al mercado, elaboración y experiencia en casos de negocio.

2. Contratación por honorarios de personal para la elaboración de los trabajos y coordinación de los mismos con empresas expertas en el tema y que a su vez puedan elaborar trabajos de inteligencia estratégica para la red.

El representante académico será quién proponga al comité técnico académico a la o las empresas para su aprobación y el reporte de los trabajos elaborados se presentarán al representante académico de la red y al comité técnico académico. 
Los investigadores participantes de la red sólo podrán ser admitidos a través de la convocatoria que emita la dirección de redes y sean evaluados positivamente por el comité evaluador de la red.

El comité técnico académico dará su respuesta en la siguiente sesión de revisión de resultados y el Investigador firmará un convenio de adhesión a la red en la que se compromete a cumplir con el manual interno de operación.

\section{ACCIONES DE INICIO EN 2009}

Para el inicio de las acciones de trabajo de la red se llevaron a cabo las siguientes acciones:

1. Se construyó una página electrónica (nanored.org.mx) que sirve como un vínculo de comunicación con diferentes sectores como el científico, educativo, el gubernamental, el empresarial y el social a través de una página electrónica interactiva diseñada por expertos. Se da mantenimiento constante a la red para que contenga información científica y tecnológica de actualidad relativa a los avances del país y especialmente de material que refleje el impacto de la nanociencia y la nanotecnología.

2. Se organizó un taller temático el 27 y 28 de marzo de 2009 en la ciudad de Puebla, intitulado "Taller de la Red de nanociencias y nanotecnología" con 50 expertos nacionales y 5 internacionales. Se presentaron los principales grupos de investigación y se analizaron las posibles interacciones entre los diferentes grupos para establecer prioridades nacionales explotando las fortalezas de los participantes y disminuyendo las debilidades.

3. Con la finalidad de incorporar al sector empresarial a la red, se organizó una reunión con empresarios en la ciudad de Monterrey, el 9 de septiembre de 2009, para definir la estrategia que permita que la red se involucre con este sector. La nanotecnología requiere de la vinculación con las empresas nacionales.

4. Se contrató a una compañía experta que realizó una prepropuesta de organización y administración de la red para poder tener desde el inicio un instrumento que permita el funcionamiento eficiente de la red.

5. Se realizó un video informativo dirigido al público en general que busca extender el conocimiento sobre Nanociencia y Nanotecnología en México y sus alcances. Con este video se busca reforzar desde un inicio el vínculo informativo con la sociedad, además de de la página electrónica.

6. Se organizaron reuniones del comité técnico académico de la red para dar seguimiento a las acciones de arranque.

\section{ACTIVIDADES DE LA RED DE NANOCIENCIAS Y NANOTECNOLOGÍA EN 2010}

\section{Reuniones del comité técnico académico de la red}

En el transcurso del 2010 se han realizado 9 reuniones, de las cuales 7 han sido por medio de videoconferencias y 2 de manera presencial. En dichas reuniones se resuelven los casos administrativos, presupuestales y de logística que conlleva el manejo de 
esta red, se promueve la labor conjunta de los miembros del comité para el fortalecimiento y reconocimiento de esta nueva área de investigación y se establecen los medios por los cuales se otorgarán los recursos destinados para la investigación.

Página de la red de nanociencias y nanotecnología

La red cuenta con una página electrónica, en la cual se puede encontrar información acerca de:

- Miembros del comité técnico académico

- Líneas de investigación en la que trabajan los miembros de la red

- Eventos relacionados con las nanociencias

- Avisos importantes para la comunidad científica

Esta página fue creada desde el 2009.

Miembros de la red de nanociencias y nanotecnología

Universidad Nacional Autónoma de México $\quad 51$

Centro de Investigación en Materiales Avanzados $\quad 16$

$\begin{array}{lr}\text { Centro de Investigaciones en Óptica } & 10\end{array}$

Benemérita Universidad Autónoma de Puebla $\quad 6$

Universidad Autónoma de Nuevo León $\quad 6$

Centro de Investigación en Alimentación y Desarrollo 4

Dirección General de Educación Superior Tecnológica - Cancún 3

Universidad Autónoma de Chihuahua 3

Universidad de Guadalajara 3

Universidad de Sonora 3

Instituto Nacional de Investigaciones Nucleares $\quad 2$

Instituto Potosino de Investigación Científica y Tecnológica 2

IPN / Centro de Investigación y de Estudios Avanzados, Querétaro 2

Universidad Autónoma de San Luis Potosí 2

Universidad Autónoma Metropolitana 2

Universidad Veracruzana / Dirección General de Investigaciones 2

Centro de Investigación y Asistencia en Tecnología y Diseño del Estado de Jalisco 1

Centro de Investigación y Desarrollo Tecnológico en Electroquímica 1

CIATEC AC 1

Universidad Autónoma de Baja California (Tijuana) 1

Universidad Autónoma de Ciudad Juárez $\quad 1$

Universidad Autónoma de México 1

Universidad Autónoma del Estado de México 1

Universidad Autónoma del Estado de Morelos $\quad 1$

$\begin{array}{lr}\text { Total } & 125\end{array}$

Líneas temáticas en las que trabajan los miembros de la red

Con base en los proyectos presentados por los investigadores en el momento de su solicitud de ingreso a la red se determinaron diez áreas principales: 
Nanoestructuras y sistemas nanoestructurados

Síntesis y aplicación de nanomateriales

Cálculo teórico y modelos computacionales

Nanocatálisis y aplicaciones ambientales

Películas delgadas

Nanomedicina y nanobiotecnología

Nanopartículas y nanocompuestos poliméricos

Nanofotónica y nanoelectrónica

Aspectos sociales, éticos y de sustentabilidad

Sensores de gases

Convocatorias para el otorgamiento de recursos económicos

En el transcurso de 2010 se han lanzado 2 convocatorias para otorgamiento de recursos económicos, la primera de ellas fue lanzada en el mes de mayo y la segunda en el mes de julio. Se planea lanzar una tercera próximamente.

Los rubros de apoyo son:

- Apoyos para pasajes y viáticos (100)

- Apoyos para estancias académicas (50)

- Apoyos para becas de licenciatura (15)

- Apoyos para becas de maestría, hasta seis meses (15)

- Apoyos para becas de doctorado, hasta seis meses (15)

- Apoyos para organización eventos Académicos (15)

- Apoyos para adquisición de equipo de Laboratorio (6)

- Apoyos para Registros de propiedad intelectual (4)

De los cuales se otorgaron en la primera convocatoria:

- 40 apoyos para pasajes y viáticos

- 20 apoyos para estancias académicas

- 10 apoyos para becas de licenciatura

- 5 apoyos para becas de maestría

- 4 apoyos para becas de doctorado

- 10 apoyos para eventos académicos

- 1 apoyo para adquisición de equipo de laboratorio

- 0 apoyo para el registro de la propiedad intelectual

En la segunda convocatoria se otorgaron:

- 33 apoyos para pasajes y viáticos

- 11 apoyos para estancias académicas

- 15 apoyos para beca de licenciatura

- 14 apoyos para beca de maestría

- 4 apoyos para beca de doctorado

- 0 apoyo para eventos académicos

- 1 apoyo para registro de la propiedad intelectual 
* El número de becas otorgadas aumentó, pues no en todos los casos se solicitaron los 6 meses y al ser menor el tiempo solicitado, se abrió la posibilidad de otorgar más becas.

Está en proceso de decidirse el apoyo para la compra de equipo.

\section{Procesos sustantivos}

Los procesos sustantivos son los relacionados directamente con la razón de ser de la organización y son importantes tanto para la estrategia global como para los clientes y los logros de la red.

a. Integración, presentación y aprobación de proyectos

Diagrama de PROCESO

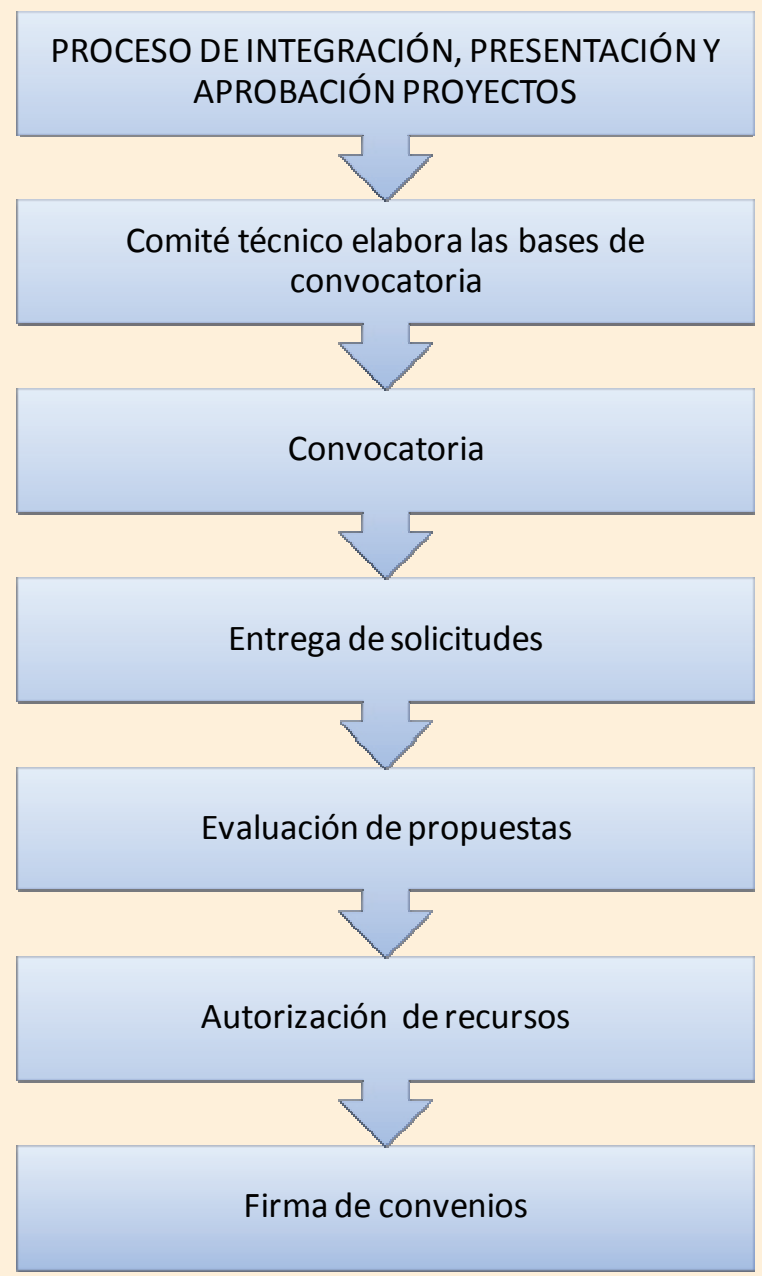




\section{DESCRIPCIÓN}

\section{Convocatorias de propuestas}

Durante el año el comité técnico realizará una convocatoria para la presentación de propuestas a la red temática, con el fin de ser evaluadas por el comité técnico académico y revisadas en conjunto con la planeación estratégica alineada a los temas definidos como prioritarios por la red, con la finalidad de poder ser autorizados de acuerdo con los criterios definidos por el Conacyt.

\section{Las propuestas de proyectos}

El paquete de información relevante de la convocatoria para las propuestas describe el contenido de una propuesta.

A fin de ayudar a simplificar la propuesta, la información proporcionada debe ser sólo de suficiente «nivel de gestión» detallada como para permitir la evaluación objetiva del merito científico técnico de la propuesta y de los recursos que se emplearán. Más detalles pueden ser solicitados según sea necesario durante la evaluación y las fases de negociación.

Además, a los solicitantes se les pedirá que sólo proporcionen una descripción resumida de las actividades para la duración completa del proyecto.

En el momento de presentar su propuesta, los participantes deben tener al menos colectivamente el potencial de recursos (humanos y materiales) necesarios para llevar a cabo el proyecto.

\section{ETAPA DOS PRESENTACIÓN DE LA PROPUESTA}

La red temática también podrá optar por organizar sus convocatorias de propuestas en dos etapas donde, en la primera, es presentado un esbozo de propuesta de los aspectos esenciales del proyecto y es evaluado con la ayuda de expertos externos; una propuesta completa se presenta en un segunda etapa de los preaprobados después de la evaluación inicial. Cuando se va a seguir un enfoque en dos fases, éste será especificado en la convocatoria.

\section{Presentación de proyectos}

La solicitud de recursos seguirá el orden siguiente:

El Investigador presentará su solicitud de recursos dentro de las fechas señaladas en el sistema establecido para ese propósito, en la página electrónica de la RNyN. Las solicitudes serán evaluadas por el CTA, con base en la calidad viabilidad y pertinencia de los proyectos presentados. Los proyectos deberán estar alineados a la visión y estrategia general de la red. La autorización de recursos la hará el comité técnico académico (comité de I+D y evaluación de proyectos) y la entrega de los mismos será responsabilidad del representante académico apoyado en el despacho externo.

El apoyo será de la siguiente forma:

Transferencias financieras por cada ministración solicitada de acuerdo con la política definida por Conacyt. 


\section{Evaluación de propuestas}

Los principios fundamentales que rigen la evaluación de las propuestas son los siguientes:

- Calidad: los proyectos para ser financiados deben demostrar un alto nivel científico, técnico y de gestión de calidad en el contexto de los objetivos del programa específico en cuestión.

- Enfoque: orientación a la planeación estratégica con enfoque a los sectores de mercado definidos y a la definición de proyectos I+D e I+D+i.

- Transparencia: con el fin de proporcionar un marco claro para los investigadores, la preparación de propuestas de financiación y para la evaluación de las propuestas evaluadoras, el proceso de llegar a las decisiones de financiación debe estar claramente descrito y estar a disposición de cualquiera de las partes interesadas. Además, la debida información debe ser proporcionada a los proponentes sobre los resultados de la evaluación de sus propuestas.

- Igualdad de trato: todas las propuestas deberán ser tratadas por igual, independientemente de su lugar de origen o la identidad de los proponentes.

- Imparcialidad: todas las propuestas deberán ser tratadas con imparcialidad en sus méritos.

- Eficacia y rapidez: los procedimientos deberán ser diseñados para ser lo más expeditos, manteniendo la calidad de la evaluación, para ser rentables y respetar el marco jurídico dentro del programa específico.

- Consideraciones éticas: cualquier propuesta que contravenga los principios éticos fundamentales podrá ser excluida de ser evaluada o seleccionada en cualquier momento.

\section{CRITERIOS de eVAluación}

Las iniciativas y proyectos de investigación y desarrollo se integrarán de acuerdo con dos lineamientos.

1. Proyectos de I+D que tengan un avance significativo para lograr su aplicación en temas prioritarios definidos por la red.

2. Proyectos generados de $\mathrm{I}+\mathrm{D}$ a partir de las oportunidades detectadas en el ámbito nacional y que sean tema prioritario para la Red.

Las propuestas se deberán integrar con las siguientes etapas:

1. Conceptualización.

2. Diseño.

3. Implementación.

En cada etapa se deberán especificar los recursos humanos y de infraestructuras requeridos, el monto de gasto y el tiempo de consecución así como los resultados esperados por etapa, tanto humanos como técnicos y los factores clave para su seguimiento y evaluación. 
El siguiente conjunto básico de criterios está destinado a ser común a todos los temas prioritarios para la evaluación de las propuestas de proyectos integrados. Estas cuestiones serán detalladas y complementadas, en caso necesario, en las convocatorias de propuestas.

- Relevancia de los objetivos en la medida en que:

- El proyecto propuesto se direccione a los objetivos del programa de trabajo en las áreas abiertas para la convocatoria.

- Posibles consecuencias en la medida en que:

- El proyecto es ambicioso adecuadamente en términos de su posición estratégica en el fortalecimiento de la competitividad o en la solución de los problemas nacionales;

— Las actividades relacionadas con la innovación y la explotación o difusión de los planes son suficientes para garantizar un uso óptimo de los resultados del proyecto.

- Excelencia en la medida en que:

- El proyecto tiene objetivos claramente definidos.

— Los objetivos representan un claro avance más allá del estado actual del arte

- La propuesta permite que el proyecto alcance sus objetivos en la investigación y la innovación.

- Calidad de la red temática en la medida en que:

— Los participantes constituyen colectivamente una red de alta calidad.

- Los participantes se adaptan bien y se comprometen con las tareas asignadas.

- Existe una buena complementariedad entre los participantes.

- Los perfiles de los participantes, incluidos los que se añadiran más adelante, han sido claramente descritos.

- Calidad de la gestión en la medida en que:

— La estructura de la organización está bien adaptada a la complejidad del proyecto y el grado de integración necesario.

— La gestión del proyecto es demostrablemente de alta calidad.

- Existe un plan satisfactorio para la gestión del conocimiento, de la propiedad intelectual y de otras actividades relacionadas con la innovación.

- Movilización de los recursos en la medida en que:

- El proyecto moviliza la masa crítica de recursos (personal, equipo, finanzas, entre muchos otros) necesarios para el éxito;

- Los recursos son integrados de manera adecuada para formar un proyecto coherente

— El plan de financiación para el proyecto es adecuado. 
Además de los criterios mencionados, así como las interpretaciones de los criterios especificados en la correspondiente convocatoria de propuestas, las siguientes preguntas también se abordarán en cualquier momento adecuado en la evaluación:

- ¿Hay ética o cuestiones de seguridad relacionadas con el tema de la propuesta? En caso afirmativo, ¿se han tenido debidamente en cuenta en la preparación de la propuesta? ¿La investigación es compatible con los principios éticos fundamentales, en su caso? Antes de que sean seleccionadas para financiación, todas las propuestas que se ocupan de cuestiones éticas sensibles y cualquier propuesta de preocupaciones éticas que se hayan identificado durante la evaluación científica podrán ser revisadas por un panel de revisión ética.

- ¿Hasta qué punto la propuesta demuestra una disposición a entablar con los actores más allá de la comunidad investigadora un público en su conjunto, para ayudar a difundir la conciencia y el conocimiento y, asimismo, explorar las repercusiones sociales más amplias del trabajo propuesto?

- Disponer de sinergias con todos los niveles de educación (en su caso) ¿están claramente establecidos?

\section{Autorización de ReCursos}

Los proyectos aprobados serán presentados al área definida por el Conacyt por parte del representante académico para su captura y procedimientos necesarios con el fin de entregar los recursos a los investigadores de acuerdo con el proceso aprobado y las normas de operación del despacho externo que controla la revisión y entrega de los recursos.

Los participantes se comprometen a cumplir con las auditorías, revisiones y todos los requisitos solicitados por el Conacyt y el o los despachos externos asignados para el control de los recursos. 